\title{
Robot-Assisted Radical Prostatectomy in Patients With Clinically High-Risk Prostate Cancer: Surgical Feasibility and Long-Term Functional and Oncologic Outcomes
}

\author{
Yong Seong Lee ( $\nabla$ novavia@hallym.or.kr) \\ Hallym University Sacred Heart Hospital https://orcid.org/0000-0003-0611-4554 \\ Tae Young Shin \\ Ewha Women's University Mokdong Hospital
}

\section{Research}

Keywords: High risk, Prostate cancer, Robot-assisted radical prostatectomy, Positive surgical margins, Biochemical recurrence, Clinical recurrence

Posted Date: April 27th, 2021

DOI: https://doi.org/10.21203/rs.3.rs-425802/v1

License: (c) (i) This work is licensed under a Creative Commons Attribution 4.0 International License. Read Full License 


\section{Abstract \\ Background}

Robot-assisted radical prostatectomy (RARP) is an acceptable procedure for localized prostate cancer. However, RARP has not been offered to patients with high-risk prostate cancer. We report long-term functional and oncologic outcomes of patients who underwent RARP for clinically high-risk prostate cancer and to assess the role of RARP in patients with high-risk prostate cancer.

\section{Methods}

This study included 90 patients with high-risk prostate cancer according to the D'Amico criteria who underwent RARP between January 2014 and December 2019. High risk was based on the presence of a clinical stage of $\geq T 2 c$, a pretreatment prostate-specific antigen level $>20 \mathrm{ng} / \mathrm{mL}$, or a biopsy Gleason score $\geq 8$. Functional outcomes including postoperative continence and potency were assessed at 1, 3, 6 , and 12 months after RARP. Oncologic outcomes comprised positive surgical margins (PSMs), biochemical recurrence (BCR), BCR-free survival, and clinical recurrence (CR)-free survival rates at 1 and 3 years.

\section{Results}

The median operative time was 185 (interquartile range [IQR], 140-250) minutes. Based on postoperative pathology, the rates of PSMs in the entire cohort and in those with stage pT2 disease were $27.8 \%$ and $8.9 \%$, respectively. The continence and potency rates at 12 months were $87.8 \%$ and $56.7 \%$, respectively. The BCR rate was $23.3 \%$, and the median time to BCR was 10.5 (IQR, 3.5-26.9) months. The 1- and 3year BCR-free survival rates were $91.5 \%$ and $85.5 \%$, respectively, and the 1- and 3-year CR-free survival rates were $97.5 \%$ and $90.8 \%$, respectively.

\section{Conclusions}

Most patients with clinically high-risk prostate cancer treated with RARP remained BCR-free and CR-free during the long-term follow-up. The optimal functional and oncologic outcomes indicating RARP as a safe and feasible approach in the present study should be confirmed in future studies.

\section{Background}

Prostate cancer is the most common non-cutaneous malignancy in males in the USA and the second leading cause of cancer-related deaths in males [1, 2]. Following the implementation of early screening and prostate biopsy, most patients are diagnosed with organ-confined prostate cancer, which is potentially curable [3]. In patients with localized prostate cancer, robot-assisted radical prostatectomy 
(RARP) has largely replaced open radical prostatectomy as the standard surgical treatment [4]. However, $20-30 \%$ of patients with prostate cancer at initial diagnosis harbor clinically high-risk disease with locally advanced pathology, defined as D'Amico high-risk disease [5-7]. Due to the underlying aggressive pathology, patients with high-grade prostate cancer and a Gleason score 8-10 may subsequently experience disease recurrence, which may result in early metastasis with significant morbidity and eventual mortality; up to $85 \%$ of these patients die of prostate cancer within 10 years of diagnosis [8]. Therefore, optimal treatment approaches for high-risk prostate cancer remain controversial and there are currently no standard treatments. Many surgeons are reluctant to perform RARP in patients with clinically high-risk prostate cancer, given the relative novelty of the procedure [9]. Specifically, worse oncologic and functional outcomes are anticipated after radical prostatectomy in patients with high-risk prostate cancer. Thus, these patients are usually offered radiotherapy in combination with hormonal therapy, which is however an incomplete therapeutic approach with significant side effects and no proven survival benefits $[10,11]$.

Recently, several studies have described reasonable oncologic outcomes and survival advantages with radical prostatectomy as first-line therapy in patients with high-risk prostate cancer $[12,13]$. Thus, the European Urology Association guidelines state that radical prostatectomy can be offered as a first-line therapy in patients with high-risk prostate cancer as part of multimodality treatment [14].

The objective of the present study was to evaluate long-term functional and oncologic outcomes at one and three years after surgery in patients with clinically high-risk prostate cancer who underwent primary RARP.

\section{Methods}

This study included a retrospective analysis of 90 patients who presented with D'Amico high-risk prostate cancer and treated with RARP by a single experienced surgeon (> 1000 RARPs) between January 2014 and December 2019. High-risk prostate cancer was defined using the D'Amico classification based on a pretreatment prostate-specific antigen (PSA) $>20 \mathrm{ng} / \mathrm{mL}$, a biopsy Gleason score $\geq 8$, or clinical stage $\geq$ cT2c [7]. All patient underwent preoperative multiparametric magnetic resonance imaging to determine the location and distribution of prostate cancer.

The study was approved by the Hallym University Sacred Heart Hospital Ethics Committee. Written informed consent was obtained from all patients. Data were collected in a customized database and analyzed. All methods were carried out in accordance with relevant guidelines and regulations. We assessed following demographic data: age, body mass index, American Society of Anesthesiologists score, prostate volume, PSA level, and biopsy Gleason score. Comorbidities were assessed using the ageadjusted Charlson comorbidity index scoring system [15]. Baseline sexual function before RARP was assessed using the Sexual Health Inventory for Men questionnaire (SHIM) questionnaire, and preoperative continence was evaluated using the International Prostate Symptom Score (IPSS). Postoperative complications were recorded and evaluated using the Clavien-Dindo classification [16]. 
Primary endpoint was postoperative long-term 1-3 years functional and oncologic outcomes after RARP. At $1,3,6$, and 12 months after RARP, we evaluated potency rates using the SHIM questionnaire and continence rates using a daily pad-weighing test. Postoperative return of erectile function was scored as $\geq 4$ on question 2 of the SHIM questionnaire or the ability to have successful sexual intercourse. Patients with zero pad use per day were considered. The following pathologic variables after RARP were also evaluated: pathologic stage, Gleason score, and positive surgical margins (PSMs). Oncologic outcomes comprised biochemical recurrence (BCR), BCR-free survival, and clinical recurrence (CR)-free survival rates at one and three years after RARP. According to American Urological Association guidelines, BCR was defined as two consecutive PSA values $\geq 0.2 \mathrm{ng} / \mathrm{mL}$ [17]. CR was defined as local recurrence and/or distant metastasis confirmed by histology and/or imaging. Salvage therapy was defined as the implementation of radiotherapy or hormonal therapy $>6$ months following RARP. Patients who underwent salvage therapy were categorized to have developed BCR. Adjuvant therapy was defined as the implementation of radiotherapy or hormonal therapy within six months following RARP in the absence of BCR.

Continuous variables are reported as medians with interquartile ranges (IQRs) and categorical variables as percentages. Continence, potency, BCR-free survival, and CR-free survival after RARP were analyzed using the Kaplan-Meier method with the log-rank test. All statistical analyses were performed using SPSS Statistics for Windows version 26.0 (IBM, Armonk, NY, USA).

\section{Results}

The baseline demographic, clinical, and pathologic data of the 90 patients included in the study are summarized in Table 1. The median operative time was 185 (IQR, 140-250) minutes, the estimated blood loss was 200 (IQR, 150-450) mL, and no patient experienced intraoperative complications. Pelvic lymphadenopathy was present in 5 of the 90 patients (5.6\%). The catheter was removed 1 week after surgery in all patients. The overall postoperative Clavien-Dindo grade I-II complication rate was $8.9 \%$. Within the first year after RARP, no patient developed Clavien-Dindo grade $\geq$ III complications such as lymphocele and urinary retention, which would require additional intervention. 
Table 1

Characteristics of 90 patients with clinically high-risk prostate cancer

Parameters $\mathrm{n}=\mathbf{9 0}$

Age, median (IQR), year

$62.5(52.25-78.0)$

BMI, median (IQR), $\mathrm{kg} / \mathrm{m}^{2}$

$25.4(23.6-28.5)$

ASA score, median (IQR)

$2.0(1.0-2.0)$

PSA, median (IQR), $\mathrm{ng} / \mathrm{ml}$

$26.75(11.5-150.8)$

Prostate volume, median (IQR), cc

$38.6(23.5-98.0)$

Biopsy Gleason score

$-6$

$2(2.2 \%)$

$-7$

$28(31.1 \%)$

$-8$

39 (43.3\%)

$-\geq 9$

$21(23.3 \%)$

Clinical stage

$-\mathrm{T} 2 \mathrm{c}$

$26(28.8 \%)$

- T3a

35 (38.9\%)

$-\geq T 3 b$

$29(32.2 \%)$

SHIM score, median (IQR)

$16.5(7-24)$

IPSS score, median (IQR)

$14.5(4-28)$

Charlson comorbidity index

$-0$

$7178.9 \%)$

$-1-2$

$17(18.9 \%)$

$-\geq 3$

$2(2.2 \%)$

$I Q R$ interquartile range, $B M /$ body mass index, $A S A$ American Society of Anesthesiologists, $P S A$ prostate-specific antigen, SHIM Sexual Health Inventory for Men, IPSS International Prostate Symptom Score

The continence rates were $54.4 \%, 68.9 \%, 77.8 \%$, and $87.8 \%$ and the potency rates were $11.1 \%, 18.9 \%$, $38.9 \%$, and $56.7 \%$ at $1,3,6$, and 12 months after RARP, respectively (Table 2). Figure 1 shows the KaplanMeier curve for continence recovery (a) and potency recovery (b). All patients received penile rehabilitation with regular use of oral phosphodiesterase type 5 inhibitors, starting one week after RARP until recovery of erectile function. The median time to continence and potency recovery was 2.3 (IQR, $0.5-12.5)$ and 6.5 (IQR, 3.2-15.5) months, respectively. 
Table 2

Data on continence and potency recovery during 1-year follow-up

\begin{tabular}{|lll|}
\hline Time & Patients achieving continence, $\mathbf{n}(\%)$ & Patients achieving potency, $\mathbf{n}(\%)$ \\
\hline 1 month & $49(54.4 \%)$ & $10(11.1 \%)$ \\
\hline 3 months & $62(68.9 \%)$ & $17(18.9 \%)$ \\
\hline 6 months & $70(77.8 \%)$ & $35(38.9 \%)$ \\
\hline 12 months & $79(87.8 \%)$ & $51(56.7 \%)$ \\
\hline
\end{tabular}

Table 3 shows the histopathologic findings and follow-up data. Briefly, 28 of the 90 patients $(31.1 \%)$ presented with stage pT2 cancer, indicating organ-confined disease. Among the remaining 62 patients (68.9\%) with stage pT3 cancer based on final pathological examination, extraprostatic extension (stage pT3a) and seminal vesicle invasion (pT3b) were found in $36(40 \%)$ and $26(28.9 \%)$ patients, respectively. Additionally, 39 (43.3\%), 35 (38.9\%), and 16 (17.8\%) patients had postoperative Gleason score of 7, 8, and $\geq 9$, respectively. In the present study, 60 patients $(66.7 \%)$ had a preoperative biopsy Gleason score $\geq 8$ whereas 51 patients $(56.7 \%$ ) had a postoperative pathologic Gleason score $\geq 8$. Therefore, pathologic downgrading in the final pathologic examination was observed in $10 \%$ of the patients with high-risk prostate cancer. Finally, 43 of the 90 patients $(47.8 \%)$ did not need secondary therapy such as radiotherapy or hormonal therapy during the 3-year follow-up period. 
Table 3

Intraoperative, histopathologic, and postoperative data of 90 patients with high-risk prostate cancer undergoing RARP

\begin{tabular}{|c|c|}
\hline Parameters & $\mathrm{n}=90$ \\
\hline Operative time, median (IQR), minutes & $185(140-250)$ \\
\hline Blood loss, median (IQR), ml & $200(150-450)$ \\
\hline Blood transfusion & $1(1.1 \%)$ \\
\hline \multicolumn{2}{|l|}{ PLND } \\
\hline - extended PLND & $83(92.2 \%)$ \\
\hline - limited PLND & $7(7.8 \%)$ \\
\hline - nodal involvement & $5(5.6 \%)$ \\
\hline \multicolumn{2}{|l|}{ Complications } \\
\hline - Clavien grade I, II & $8(8.9 \%)$ \\
\hline - Clavien grade $\geq$ III & 0 \\
\hline \multicolumn{2}{|l|}{ Pathologic stage } \\
\hline - pT2 & $28(31.1 \%)$ \\
\hline - pТЗа & $36(40 \%)$ \\
\hline - pT3b & $26(28.9 \%)$ \\
\hline \multicolumn{2}{|l|}{ Pathologic Gleason score } \\
\hline-7 & $39(43.3 \%)$ \\
\hline-8 & $35(38.9 \%)$ \\
\hline$-\geq 9$ & $16(17.8 \%)$ \\
\hline \multicolumn{2}{|l|}{ PSMs } \\
\hline - overall & $25(27.8 \%)$ \\
\hline - in pT2 cancer & $8(8.9 \%)$ \\
\hline - in pT3 cancer & $17(18.9 \%)$ \\
\hline
\end{tabular}

$R A R P$ robot-assisted radical prostatectomy, IQR interquartile range, $P L N D$ pelvic lymph node dissection, $P S M s$ positive surgical margins, $B C R$ biochemical recurrence 


\begin{tabular}{|ll|}
\hline Parameters & $\mathrm{n}=90$ \\
\hline - overall & $35(38.9 \%)$ \\
\hline - radiotherapy & $12(13.3 \%)$ \\
\hline - hormonal therapy & $23(25.6 \%)$ \\
\hline Salvage treatment & $17(18.9 \%)$ \\
\hline - overall & $5(5.6 \%)$ \\
\hline - radiotherapy & $7(7.8 \%)$ \\
\hline - hormonal therapy & $5(5.6 \%)$ \\
\hline - radiotherapy and hormonal therapy & $51.5(13.5-83.6)$ \\
\hline Follow-up duration, median (IQR), month & $21(23.3 \%)$ \\
\hline - BCR & $10.5(3.5-26.9)$ \\
\hline - time to BCR, median (IQR) & \\
\hline $\begin{array}{l}R A R P \text { robot-assisted radical prostatectomy, IQR interquartile range, } \\
\text { dissection, } P S M \text { N }\end{array}$ positive surgical margins, BCR biochemical recurrence \\
\hline
\end{tabular}

PSMs on postoperative pathologic examination were found in 25 of the 90 patients (27.8\%). The rate of PSMs was lower (8.9\%) in patients with stage pT2 disease. Adjuvant therapy with radiotherapy and hormonal therapy was utilized in $12(13.3 \%)$ and 23 (25.6\%) patients, respectively, whereas 17 of the 90 patients (18.9\%) required salvage therapy. Salvage therapy after BCR was utilized in 5 patients $(5.6 \%)$ with radiotherapy and 7 patients (7.8\%) with hormonal therapy $>6$ months following RARP as well as 5 patients (5.6\%) with secondary radiotherapy combination with hormonal therapy following adjuvant therapy. We summarize the details of postoperative therapies administered following RARP (Fig. 2).

The median follow-up duration was 51.5 (IQR, 13.5-83.6) months. BCR occurred in 21 patients (23.3\%), and the median time to BCR was 10.5 (IQR, 3.5-26.9) months. The remaining 69 patients $(76.7 \%)$ did not experience BCR during the follow-up period. Of the 21 patients with BCR, 9 (42.9\%) and $12(57.1 \%)$ patients received adjuvant therapy and salvage therapy, respectively. The median PSA level at the time of BCR was 0.5 (IQR, 0.2-1.55) ng/mL. Figure 3 demonstrates the Kaplan-Meier estimates for BCR-free survival rates. The 1- and 3-year BCR-free survival rates were 91.5\% (95\% confidence interval (CI), 81.5$95.4 \%)$ and $85.5 \%(95 \% \mathrm{Cl}, 76.8-91.3 \%)$, respectively. All patients with BCR underwent pelvic magnetic resonance imaging, bone scintigraphy, and chest and abdominal computed tomography. One patient (1.1\%) was diagnosed with metastasis to the liver and brain at 24 months postoperatively. There were no cancer-related deaths. Of the 21 patients with BCR, 4 patients (19\%) underwent observation, 7 patients (33.3\%) were treated with hormonal therapy, 5 patients (23.9\%) exhibited persistently elevated PSA levels without evidence of metastasis and were treated with salvage pelvic radiotherapy, and 5 patients (23.9\%) following adjuvant therapy underwent secondary salvage radiotherapy and hormonal therapy. Finally, the 
1-and 3-year CR-free survival rates were 97.5\% (95\% Cl, 91.5-98.8\%) and 90.8\% (95\% Cl, 86.4-94.3\%), respectively.

\section{Discussion}

For patients with clinically localized prostate cancer and a life expectancy beyond 10 years, radical prostatectomy is the treatment of choice. Recently, more than $80 \%$ of radical prostatectomy procedures in the USA are performed with robotic assistance [18]. Recent literature reviews and meta-analyses show that RARP is associated with decreased rates of PSMs, improvements in potency and continence recovery at short-term follow-up, and shorter hospital stay compared to open and laparoscopic radical prostatectomy approaches in low- and intermediate-risk patients [19].

However, $20-30 \%$ of patients continue to present with D'Amico high-risk prostate cancer at the time of initial diagnosis [5-7]. Optimal treatment for those diagnosed with high-risk prostate cancer is controversial, and the role of radical prostatectomy for locally advanced high-risk prostate cancer remains a topic of debate due to several reasons, including discouragement of surgical management and evasion of RARP because of inexperience with the technique and potential difficulty in performing extended pelvic lymph node dissection [20]. Therefore, long-term oncologic outcomes of RARP in patients with high-risk prostate cancer remain poorly reported.

Several series have recently reported the role of RARP in patients with high-risk prostate cancer. Despite its aggressive behavior, the prognosis of high-risk prostate cancer is not uniformly poor. High-risk prostate cancer is confined to the prostate in many patients, who may experience long-term progression-free survival after radical prostatectomy. Yossepowitch et al. analyzed radical prostatectomy outcomes in 957 patients with clinically localized high-risk prostate cancer and found that the cancer was confined to the prostate in $43 \%$ of the patients [21]. Compared with low- and intermediate-risk patients, there was a 3.3fold increase in relapse hazard and higher likelihood of progression at five and ten years after radical prostatectomy. A multicenter study by Sooriakumaran et al. concluded that radical prostatectomy for patients with resectable distant metastases was safe in expert hands in the setting of meticulous patient selection [22]. The adoption of radical prostatectomy as a treatment option for high-risk prostate cancer was based on the reported overall (and disease-specific) survivals rates of $87 \%(93 \%), 70 \%(83 \%)$, and $58 \%(71 \%)$ at 5,10 , and 15 years, respectively [23].

The big question remains whether radical prostatectomy is superior to radiotherapy combined with hormonal therapy. Several studies retrospectively compared radical prostatectomy with radiotherapy. Boorijian et al. retrospectively compared outcomes between radical prostatectomy and radiotherapy combined with hormonal therapy in patients with high-risk prostate cancer and found comparable 10year cancer-free survival rates between the two groups; the authors also found that the risk of all-cause mortality was greater after radiotherapy with hormonal therapy compared to radical prostatectomy [24]. Zelefsky et al. found that cancer-free survival rates were comparable between radical prostatectomy and radiotherapy combined with hormonal therapy in patients with high-risk prostate cancer [25]. In that 
study, the absolute benefit of $7.8 \%$ in distant metastasis-free survival favored radical prostatectomy. Radical prostatectomy is therefore superior to radiotherapy combined with hormonal therapy in healthy patients with long life expectancy. Additionally, Boris et al. demonstrated the feasibility and durability of salvage RARP after failed radiotherapy and reported that the functional and oncologic outcomes of RARP were not inferior to those of open radical prostatectomy [26]. Based on these results, we suggest that, when feasible, RARP should be considered in patients with organ-confined high-risk prostate cancer if the patient accepts the surgical risk. Salvage RARP is another good option for the treatment of patients with organ-confined high-risk prostate cancer after failed radiotherapy.

In the present study, the median operative time was 185 minutes and the median intraoperative blood loss was $200 \mathrm{~mL}$; only one patient with cardiovascular disease received intraoperative blood transfusion. These findings are not substantially different than those reported in a systematic review of RARP outcomes in patients with high-risk prostate cancer [6]. The study reported that the mean operative time was $168 \mathrm{~min}$, the estimated blood loss was $189 \mathrm{~mL}$, the mean length of hospital stay was 3.2 days, and the duration of catheterization was 7.8 days. In the present study, the median duration of hospital stay and urinary catheter indwelling was seven days due to nature of the Korean medical insurance. Advantages of these long hospitalizations include the prevention of postoperative complications such as ileus and lymphocele.

The continence rates in the current study were consistent with the findings of other study that demonstrated the benefits of early recovery of urinary continence in patients with low- or intermediate-risk prostate cancer undergoing RARP, such as a report by Student et al. who found similar continence rates $(62.5 \%, 68.8 \%, 75.0 \%$, and $86.7 \%$ at $1,2,6$, and 12 months, respectively) [27]. We performed the detrusorrhaphy technique which is designed for thickening and strengthening the detrusor muscles from the posterior bladder neck to the bilateral dissected pedicles area; this technique is thought to prevent hyper-mobilization of the bladder neck area, thereby reducing stress urinary incontinence, and is considered important for continence recovery as we previously reported [28]. Furthermore, using a validated sexual function questionnaire, we found that the rate of return baseline preoperative sexual function scores was $11.1 \%$ one month after RARP, which subsequently increased to $18.9 \%, 38.9 \%$, and $56.7 \%$ at 3,6 , and 12 months after RARP, respectively. These outcomes are better than those reported by other studies using the same validated questionnaire in patients undergoing RARP. In their study examining nerve-sparing in salvage RARP, Bonet et al. reported that the 12-month potency rate was $25.6 \%$ in the good nerve-sparing group and that good nerve-sparing tended to be predictive of potency after salvage RARP [29]. In the current study, we performed athermal clipless intrafascial nerve-sparing technique if indicated; this technique might be associated with improved viable tissue preservation within the neurovascular bundles as we previously reported [28].

In the present study, the rate of patients with stage pT2 organ-confined prostate cancer by postoperative pathologic assessment was $31.3 \%$, similar to that reported in the systematic review of RARP-related outcomes by Yuh et al., who found that the average rate of organ-confined disease was 35\% (range, 748\%) [6]. During the 3-year follow-up period in the present study, 43 of the 90 patients (47.8\%) did not 
require secondary therapy such as radiotherapy or hormonal therapy. The rate of PSMs in the current study was $27.8 \%$, in line with previous reports, as revealed in the systematic review by Yuh et al., in which the rate of PSMs was 35\% (range, 12-53\%) [6]. The rate of PSMs was reduced to $8.9 \%$ in the patients with stage pT2 disease. In the present study, the median follow-up duration was 51.5 months. Of the 21 patients (23.3\%) who experienced BCR at a median of 10.5 months after RARP, 9 and 12 patients received adjuvant therapy and salvage therapy, respectively. Comparable to the present study findings, Kumar et al. reported that the overall BCR rate was $19.2 \%$ during a mean follow-up duration of 24.3 months and that the mean time to BCR was 7.9 months in patients with high-risk prostate cancer [30]. Our analyses revealing 3-year BCR-free and CR-free survival rates of $85.5 \%$ and $90.8 \%$, respectively, are comparable to those reported in a study of patients with high-risk prostate cancer by Rogers et al. [31].

In patients with high-risk prostate cancer, RARP should be performed by skilled and experienced surgeons rather than beginners to reduce complications and to achieve optimal surgical results, given that radical prostatectomy is associated with high morbidity. A study by Punnen et al. provides further support for the effect of surgical experience on improved outcomes with RARP in patients with high-risk prostate cancer [32].

The limitations of the present study are the retrospective and noncomparative design of the study, which was performed by a single surgeon in a single institution, and the small sample size. While the present study was not a randomized trial, we believe that the biases associated with the study design were minimal, given that the surgeon has already performed more than 1000 RARPs between 2007 and 2020 and that the surgical methods in patients with high-risk prostate cancer are not challenging. Despite the ongoing follow-up of the study patients, our initial results suggest optimal functional and oncologic outcomes with RARP in patients with high-risk prostate cancer. Future studies should be conducted to include larger cohorts with longer follow-up periods to concomitantly compare the functional and oncologic outcomes of RARP with radiotherapy and/or hormonal therapy in patients with high-risk prostate cancer in a standardized fashion.

\section{Conclusion}

RARP conferred long-term cancer control in most patients with high-risk prostate cancer and was a safe and feasible minimally invasive surgical alternative to radiotherapy or hormonal therapy in select patients with D'Amico high-risk prostate cancer. These results demonstrating the optimal functional and oncologic outcomes of RARP should be validated to assure the reproducibility of measurements in prospective randomized-controlled studies.

\section{Abbreviations}

RARP: robot-assisted radical prostatectomy; PSA: prostate-specific antigen; SHIM: Sexual Health Inventory for Men; IPSS: International Prostate Symptom Score; PSMs: positive surgical margins; BCR: biochemical recurrence; $C R$ : clinical recurrence; IQR: interquartile range 


\section{Declarations}

Acknowledgements

The authors would like to thank Enago (www.enago.co.kr) for the English language review.

\section{Authors' contributions}

TYS interpreted the patient data and was a major contribution in writing the manuscript and performed all statistical analyses of the reported data. YSL performed protocol/project development, data collection, manuscript writing, and manuscript editing. All authors read and approved the final manuscript.

\section{Funding}

The authors received no funding for this study.

\section{Availability of data and materials}

The datasets used and/or analyzed during the current study are available from the corresponding author on reasonable request.

\section{Ethics approval and consent to participate}

The Institutional Review Board of Hallym University Sacred Heart Hospital, Republic of Korea, approved this study (No. 2018-05-012).

\section{Consent for publication}

Not applicable

\section{Competing interests}

The authors declare that they have no competing interests.

\section{Author details}

${ }^{1}$ Department of Urology, Hallym University Sacred Heart Hospital, Hallym University College of Medicine, Anyang, Korea.

${ }^{2}$ Department of Urology, Ewha University College of Medicine, Seoul, Korea.

\section{References}

1. Crawford ED. Epidemiology of prostate cancer. Urology. 2003;62(6 Suppl 1):3-12. doi: 10.1016/j.urology.2003.10.013. 
2. Jani AB, Johnstone PA, Liauw SL, Master VA, Brawley OW. Age and grade trends in prostate cancer (1974-2003): a surveillance, epidemiology, and end results registry analysis. Am J Clin Oncol. 2008;31(4):375-378. doi: 10.1097/COC.0b013e3181637384.

3. Bill-Axelson A, Holmberg L, Ruutu M, et al. Radical prostatectomy versus watchful waiting in early prostate cancer. N Engl J Med. 2011;364(18):1708-1717. doi: 10.1056/NEJMoa1011967.

4. Basiri A, de la Rosette JJ, Tabatabaei S, Woo HH, Laguna MP, Shemshaki H. Comparison of retropubic, laparoscopic and robotic radical prostatectomy: who is the winner? World J Urol. 2018;36(4):609-621. doi: 10.1007/s00345-018-2174-1.

5. Cooperberg MR, Cowan J, Broering JM, Carroll PR. High-risk prostate cancer in the United States, 1990-2007. World J Urol. 2008;26(3):211-218. doi: 10.1007/s00345-008-0250-7.

6. Yuh B, Artibani W, Heidenreich A, et al. The role of robot-assisted radical prostatectomy and pelvic lymph node dissection in the management of high-risk prostate cancer: a systematic review. Eur Urol. 2014;65(5):918-927. doi: 10.1016/j.eururo.2013.05.026.

7. D'Amico AV, Whittington R, Malkowicz SB, et al. Pretreatment nomogram for prostate-specific antigen recurrence after radical prostatectomy or external-beam radiation therapy for clinically localized prostate cancer. J Clin Oncol. 1999;17(1):168-172. doi: 10.1200/JC0.1999.17.1.168.

8. Rosoff JS, Savage SJ, Prasad SM. Salvage radical prostatectomy as management of locally recurrent prostate cancer: outcomes and complications. World J Urol. 2013;31(6):1347-1352. doi: 10.1007/s00345-013-1029-z.

9. Briganti $A$, Bianchi $M$, Sun $M$, et al. Impact of the introduction of a robotic training programme on prostate cancer stage migration at a single tertiary referral centre. BJU Int. 2013;111(8):1222-1230. doi: 10.1111/j.1464-410X.2012.11464.x.

10. D'Amico AV, Chen MH, Renshaw AA, Loffredo M, Kantoff PW. Androgen suppression and radiation vs radiation alone for prostate cancer: a randomized trial. JAMA. 2008;299(3):289-295. doi: 10.1001/jama.299.3.289.

11. Tayler LG, Canfield SE, Du XL. Review of major adverse effects of androgen-deprivation therapy in men with prostate cancer. Cancer. 2009;115(11):2388-2399. doi: 10.1002/cncr.24283.

12. Connolly SS, Cathcart PJ, Gilmore P, et al. Robotic radical prostatectomy as the initial step in multimodal therapy for men with high-risk localised prostate cancer: initial experience of 160 men. BJU Int. 2012;109(5):752-759. doi: 10.1111/j.1464-410X.2011.10548.x.

13. Hou GL, Luo Y, Di JM, et al. Predictors of urinary continence recovery after modified radical prostatectomy for clinically high-risk prostate cancer. Urol J. 2015;12(1):2021-2027. doi: 10.22037/uj.v12i1.2923.

14. Heidenreich A, Bastian PJ, Bellmunt J, et al. EAU guidelines on prostate cancer. part 1: screening, diagnosis, and local treatment with curative intent-update 2013. Eur Urol. 2014;65(1):124-137. doi: 10.1016/j.eururo.2013.09.046.

15. Charlson ME, Pompei P, Ales KL, MacKenzie CR. A new method of classifying prognostic comorbidity in longitudinal studies: development and validation. J Chronic Dis. 1987;40(5):373-383. doi: 
10.1016/0021-9681(87)90171-8.

16. Dindo D, Demartines N, Clavien PA. Classification of surgical complications: a new proposal with evaluation in a cohort of 6336 patients and results of a survey. Ann Surg. 2004;240(2):205-213. doi: 10.1097/01.sla.0000133083.54934.ae.

17. Cookson MS, Aus G, Burnett AL, et al. Variation in the definition of biochemical recurrence in patients treated for localized prostate cancer: the American Urological Association Prostate Guidelines for Localized Prostate Cancer Update Panel report and recommendations for a standard in the reporting of surgical outcomes. J Urol. 2007;177(2):540-545. doi: 10.1016/j.juro.2006.10.097.

18. Oberlin DT, Flum AS, Lai JD, Meeks JJ. The effect of minimally invasive prostatectomy on practice patterns of American urologists. Urol Oncol. 2016;34(6):255.e1-5. doi:

10.1016/j.urolonc.2016.01.008.

19. Moran PS, O'Neill M, Teljeur C, Flattery M, Murphy LA, Smyth G, Ryan M. Robot-assisted radical prostatectomy compared with open and laparoscopic approaches: a systematic review and metaanalysis. Int J Urol. 2013;20(3):312-321. doi: 10.1111/iju.12070.

20. Jayram G, Decastro GJ, Large MC, Razmaria A, Zagaja GP, Shalhav AL, Brendler CB. Robotic radical prostatectomy in patients with high-risk disease: a review of short-term outcomes from a highvolume center. J Endourol. 2011;25(3):455-457. doi: 10.1111/iju.12070.

21. Yossepowitch O. Eggener SE, Bianco FJ Jr, et al. Radical prostatectomy for clinically localized, high risk prostate cancer: critical analysis of risk assessment methods. J Urol. 2007;178(2):493-499; discussion 499. doi: 10.1016/j.juro.2007.03.105.

22. Sooriakumaran P, Karnes J, Stief C, et al. A multi-institutional analysis of perioperative outcomes in 106 men who underwent radical prostatectomy for distant metastatic prostate cancer at presentation. Eur Urol. 2016;69(5):788-794. doi: 10.1016/j.eururo.2015.05.023.

23. Zwergel U, Suttmann H, Schroeder T, et al. Outcome of prostate cancer patients with initial PSA > or $=20 \mathrm{ng} / \mathrm{ml}$ undergoing radical prostatectomy. Eur Urol. 2007;52(4):1058-1065. doi: 10.1016/j.eururo.2007.03.056.

24. Boorjian SA, Karnes RJ, Viterbo R, et al. Long-term survival after radical prostatectomy versus external-beam radiotherapy for patients with high-risk prostate cancer. Cancer. 2011;117(13):28832891. doi: $10.1002 /$ cncr.25900.

25. Zelefsky MJ, Eastham JA, Cronin AM, et al. Metastasis after radical prostatectomy or external beam radiotherapy for patients with clinically localized prostate cancer: a comparison of clinical cohorts adjusted for case mix. J Clin Oncol. 2010;28(9):1508-1513. doi: 10.1200/JC0.2009.22.2265.

26. Boris RS, Bhandari A, Krane LS, Eun D, Kaul S, Peabody JO. Salvage robotic-assisted radical prostatectomy: initial results and early report of outcomes. BJU Int. 2009;103(7):952-956. doi: 10.1111/j.1464-410X.2008.08245.x.

27. Student V Jr, Vidlar A, Greple M, Hartmann I, Buresova E, Student V. Advanced reconstruction of vesicourethral support (ARVUS) during robot-assisted radical prostatectomy: one-year functional 
outcomes in a two-group randomized controlled trial. Eur Urol. 2017;71(5):822-830. doi: 10.1016/j.eururo.2016.05.032.

28. Shin TY, Lee YS. Detrusorrhaphy and intrafascial nerve-sparing during robot-assisted radical prostatectomy on recovery of continence and potency: surgical feasibility, one-year functional and oncologic outcomes. Urol J. 2020. doi: 10.22037/uj.v16i7.5915.

29. Bonet X, Ogaya-Pinies G, Woodlief T, et al. Nerve-sparing in salvage robot-assisted prostatectomy: surgical technique, oncological and functional outcomes at a single high-volume institution. BJU Int. 2018;122(5):837-844. doi: 10.1111/bju.14517.

30. Kumar A, Samavedi S, Bates AS, et al. Safety of selective nerve sparing in high risk prostate cancer during robot-assisted radical prostatectomy. J Robot Surg. 2017;11(2):129-138. doi: 10.1007/s11701-016-0627-3.

31. Rogers CG, Sammon JD, Sukumar S, Diaz M, Peabody J, Menon M. Robot assisted radical prostatectomy for elderly patients with high risk prostate cancer. Urol Oncol. 2013;31(2):193-197. doi: 10.1016/j.urolonc.2010.11.018.

32. Punnen S, Meng MV, Cooperberg MR, Greene KL, Cowan JE, Carroll PR. How does robot-assisted radical prostatectomy (RARP) compare with open surgery in men with high-risk prostate cancer? BJU Int. 2013;112(4):E314-320. doi: 10.1111/j.1464-410X.2012.

\section{Figures}
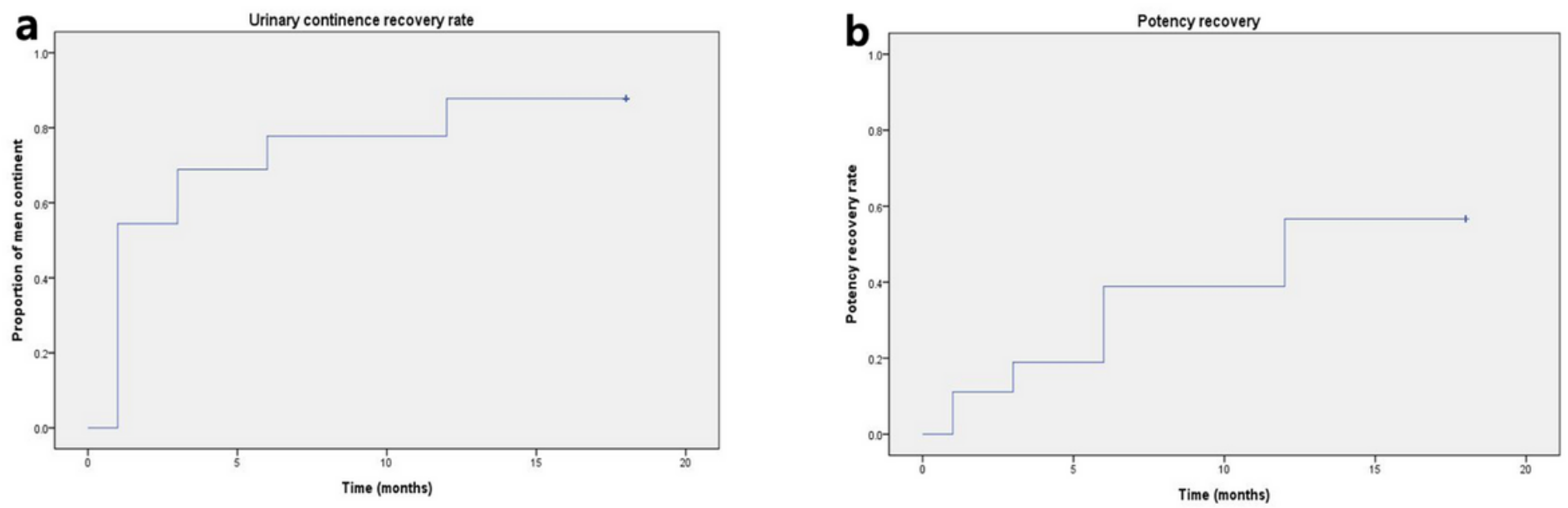

\section{Figure 1}

Kaplan-Meier curve showing estimated continence recovery (a) and potency recovery (b) 


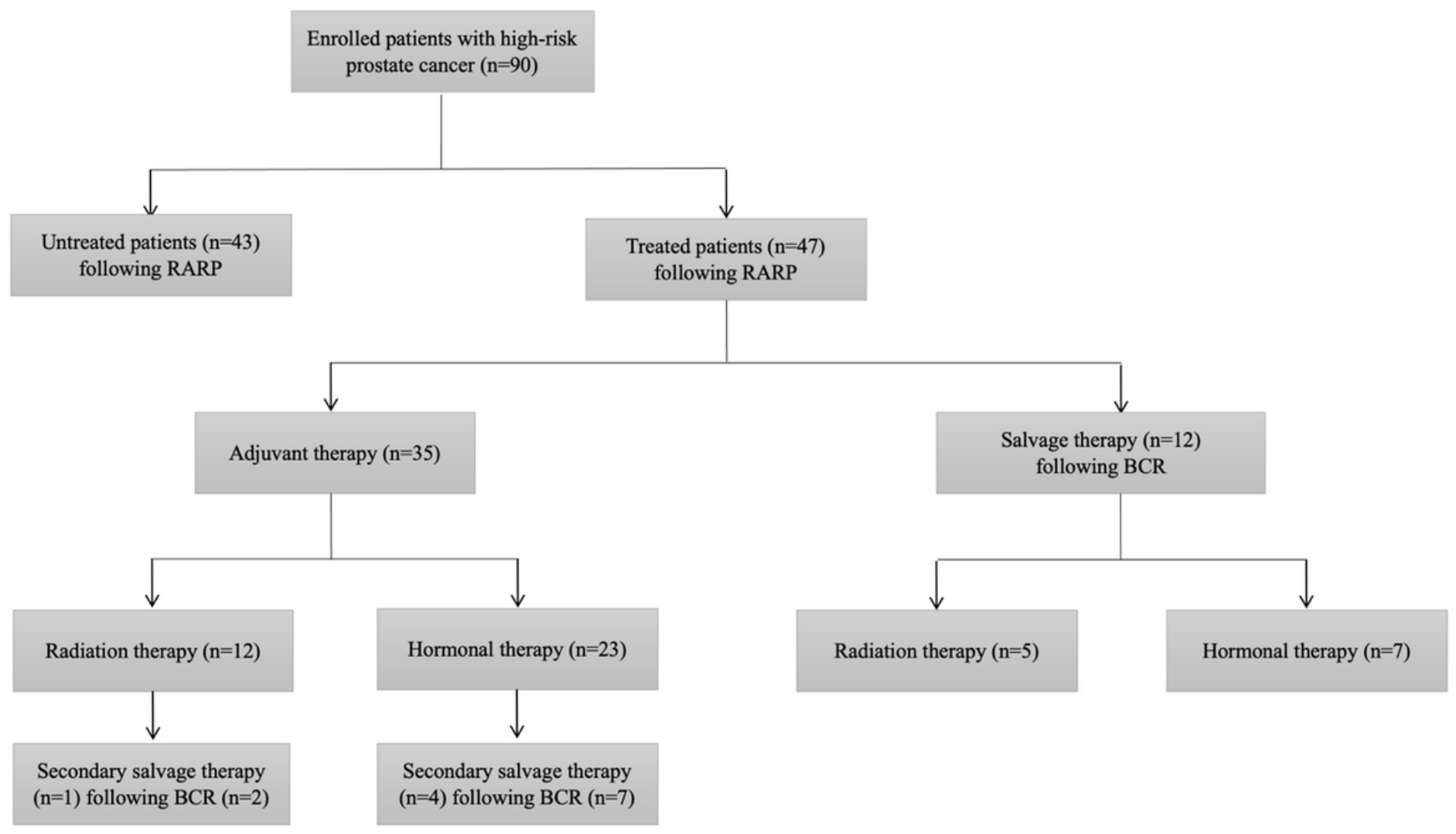

\section{Figure 2}

Treatment stratification tree based on the data of 90 patients with high-risk prostate cancer undergoing RARP RARP robot-assisted radical prostatectomy, BCR biochemical recurrence 


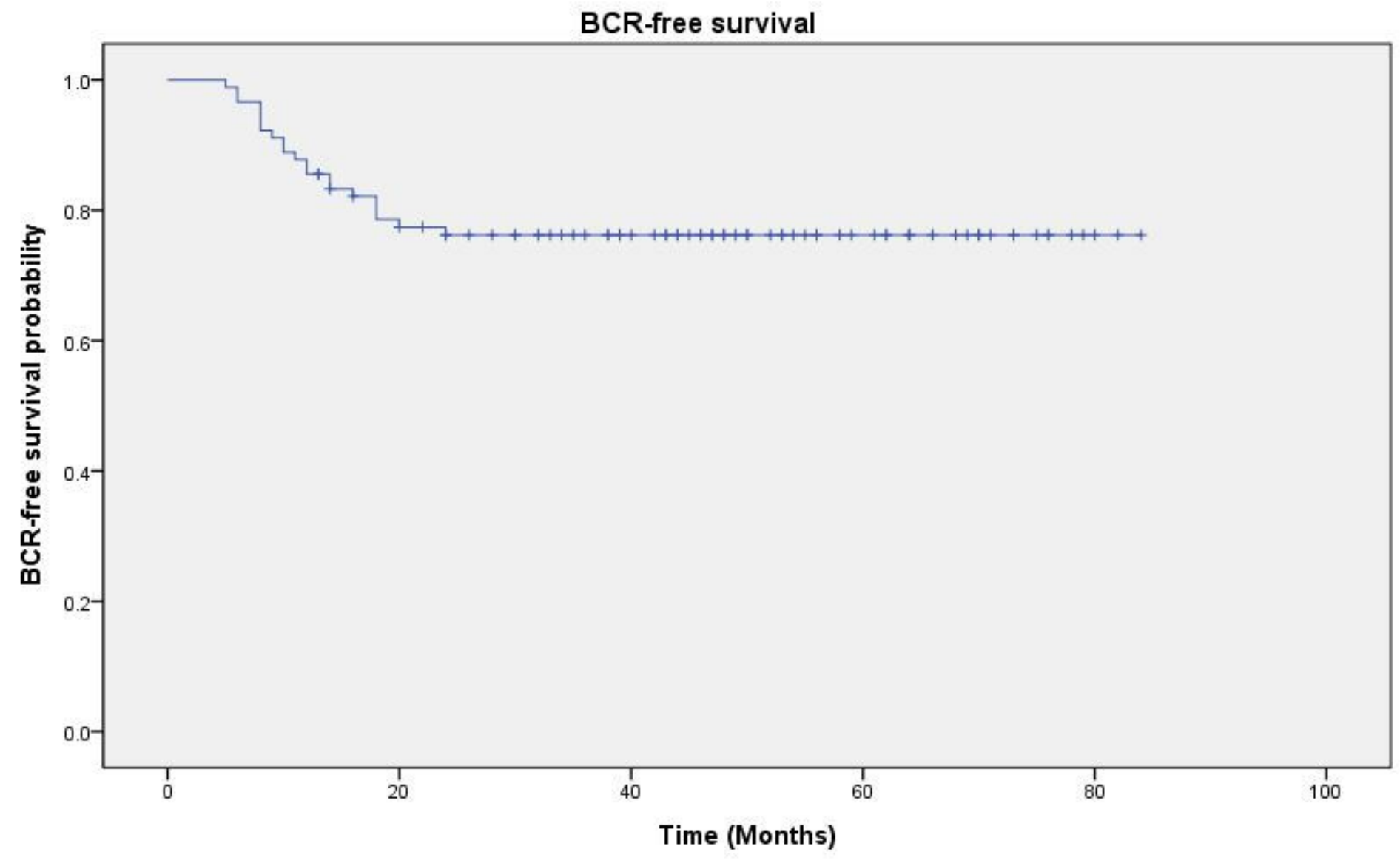

Figure 3

Kaplan-Meier curve showing estimated probability of BCR-free survival after RARP RARP robot-assisted radical prostatectomy, BCR biochemical recurrence 